\title{
Overexpression of the miR-34 family suppresses invasive growth of malignant melanoma with the wild-type p53 gene
}

\author{
HITOSHI YAMAZAKI ${ }^{1}$, TSUYOSHI CHIJIWA ${ }^{2}$, YOSHIMASA INOUE ${ }^{3}$, YOSHIYUKI ABE ${ }^{4}$, \\ HIROSHI SUEMIZU ${ }^{5}$, KENJI KAWAI ${ }^{5}$, MASATOSHI WAKUI ${ }^{6}$, DAISUKE FURUKAWA ${ }^{7}$, \\ MASAYA MUKAI $^{3}$, SADAHITO KUWAO ${ }^{8}$, MAKOTO SAEGUSA $^{1}$ and MASATO NAKAMURA ${ }^{9}$
}

\begin{abstract}
${ }^{1}$ Department of Pathology, Kitasato University School of Medicine, Kanagawa; ${ }^{2}$ Japan Self Defense Force Hospital Naha, Okinawa; ${ }^{3}$ Department of Surgery, Tokai University Hachioji Hospital, Tokyo; ${ }^{4}$ Tokorozawa PET Diagnostic Imaging Clinic, Saitama; ${ }^{5}$ Central Institute for Experimental Animals, Kanagawa; ${ }^{6}$ Department of Laboratory Medicine, Keio University School of Medicine, Tokyo; ${ }^{7}$ Department of Surgery, Tokai University School of Medicine, Kanagawa;

${ }^{8}$ Department of Pathology and Cytology, Higashi-Yamato Hospital, Tokyo;

${ }^{9}$ Regenerative Medicine and Pathology, Tokai University School of Medicine, Kanagawa, Japan
\end{abstract}

Received December 26, 2011; Accepted January 30, 2012

DOI: 10.3892/etm.2012.497

\begin{abstract}
Malignant melanoma is the most aggressive neoplasm, with severe metastatic potential. microRNAs represent a class of endogenously expressed, small non-coding RNAs that regulate gene expression. As a consequence, the translation of these mRNAs is inhibited or they are destabilized resulting in downregulation of the encoded protein. The microRNA-34 (miR-34) family, which comprises three processed microRNAs (miR-34a/b/c) was identified as the mediator of tumor suppression by p53. Many reports suggest that the miR-34s contribute to the inhibition of invasion or metastasis in various tumor types. In this study, we evaluated the expression of the miR-34 family in four human melanoma cell lines (A375, G361, C32TG and SK-MEL-24) which have the wild-type p53 gene using real-time reverse transcription PCR. We also examined their generative and invasive characteristics using the cell proliferation assay and the invasion/ migration assay, respectively. All four melanoma cell lines showed significant expression of miR-34s - A375: miR-34a 0.6176, miR-34b 0.7625, miR-34c 0.7877; G361: 7.6424, 16.4127, 22.0332; C32TG: 2.1630, 2.1091, 8.4425; SK-MEL-24: 0.3621, $2.5659,8.5907$. The cell doubling times of these cell lines in h:min were as follows: A375 23:23, G361 68:24, C32TG 47:22 and SK-MEL-24 67:03. The in vitro generation times of G361 and SK-MEL-24, which showed increased expression of miR-34c, were significantly shorter than A375 with decreased expression of miR-34c ( $\mathrm{p}=0.0063$, ANOVA). Invasion (\%) of
\end{abstract}

Correspondence to: Professor Hitoshi Yamazaki, Department of Pathology, Kitasato University School of Medicine, 1-15-1 Kitasato, Minami-ku, Sagamihara, Kanagawa 252-0374, Japan

E-mail: powder1104@hotmail.com

Key words: microRNA-34, melanoma, p53 the four cell lines was as follows: A375 44.0\%, G361 22.4\%, C32TG $13.8 \%$ and SK-MEL-24 45.0\%. In vitro invasiveness of G361 and C32TG, which showed increased expression of miR-34a, was significantly suppressed ( $\mathrm{p}=0.005$, ANOVA). These results suggest that overexpression of miR-34a and c suppresses invasive and generative potentials, respectively, in human malignant melanoma.

\section{Introduction}

Malignant melanoma is the most aggressive neoplasm with severe metastatic potential. In recent decades, the incidence of malignant melanoma has steadily increased. A particularly worrying feature of the tumor is its increasing incidence and its capacity for rapid metastatic spread. microRNAs represent a class of endogenously expressed, small non-coding RNAs that regulate gene expression $(1,2)$. As a consequence of translation, these mRNAs are inhibited or destabilized, resulting in downregulation of the encoded protein. A few microRNAs have been classified as oncogenes or tumor-suppressor genes as their expression is altered in tumors, which in some cases has been shown to contribute to the phenotypes of cancer cells. Recently, the microRNA-34 (miR-34) family was identified as the mediator of tumor suppression by p53 (1). Many reports suggest that the miR-34s contribute to inhibition of invasion or metastasis in various tumors. These facts suggest that miR-34s play an important role as inhibitors of tumor growth. However, the biological characteristics of miR-34s in human malignant melanoma are not well understood (3). In this study, we evaluated the expression of miR-34 family members in four human melanoma cell lines (A375, G361, C32TG and SK-MEL-24) which have the wild-type p53 gene using real-time reverse transcription PCR. We also examined their generative and invasive characteristics using the cell proliferation assay and the invasion/migration assay. 


\section{Materials and methods}

Cell culture. Human melanoma cell lines A375 and G361 were obtained from Dainippon Pharmaceutical Co., Ltd. (Osaka, Japan) and the Health Science Research Resources Bank (Osaka, Japan), respectively. SK-MEL-24 and C32TG were obtained from the American Type Culture Collection (Manassas, VA, USA). These four cell lines were confirmed to have the wild-type p53 gene status (4-6). Cell lines were maintained in Dulbecco's modified minimal essential medium (DMEM) supplemented with $10 \%$ heat-inactivated fetal bovine serum (FBS), $100 \mathrm{U} / \mathrm{ml}$ penicillin and $100 \mu \mathrm{g} / \mathrm{ml}$ streptomycin.

Quantitative evaluation of miR-34s. Total RNA containing microRNA was extracted using a mirVana ${ }^{\mathrm{TM}}$ miRNA Isolation Kit (Applied Biosystems, Foster City, CA, USA) and cDNA was synthesized using a TaqMan ${ }^{\circledR}$ MicroRNA Reverse Transcription Kit (Applied Biosystems). Quantitative reverse transcription PCR (RT-PCR) for $\mathrm{miR}-34 \mathrm{a} / \mathrm{b} / \mathrm{c}$ and U6 snRNA(RNU6B) was performed according to the manufacturer's recommendations. The primers for miR-34a (MI0000268), miR-34b (MI0000742), miR-34c (MI0000743) and RNU6B (NR002752) were purchased from TaqMan ${ }^{\circledR}$ MicroRNA Assays (Applied Biosystems). We used TaqMan ${ }^{\circledR}$ Universal PCR Master Mix, No AmpErase ${ }^{\circledast}$ UNG (Applied Biosystems) for the real-time PCR. Real-time RT-PCR assays were run on Thermal Cycler Dice ${ }^{\circledR}$ TP800 (Takara Bio, Inc., Japan) with the comparative $\Delta \mathrm{Ct}$ method $(7,8)$. All samples were assayed in quadruplicate and values were normalized by the respective amounts of RNU6B expression as an endogenous control. The positive control standard was T5, a thrombospondin-2-overexpressing human melanoma cell line established by our laboratory (9).

Growth analyses of the cell lines. The cells were seeded at $1.0 \times 10^{3}$ cells/well in $2-\mathrm{cm}$ dishes. The cell number was determined with a Coulter Counter (Beckman Coulter, Fullerton, CA). Quadruplicate cultures of each cell line were prepared at all time points (10).

In vitro invasion/migration assays. Cell invasion was assayed in BD BioCoat ${ }^{\mathrm{TM}}$ Matrigel $^{\mathrm{TM}}$ Invasion Chambers (24-well, $8-\mu \mathrm{m}$ pore, Becton-Dickinson Labware, Bedford, MA, USA). Control insert chambers were used for migration assays. DMEM supplemented with 5\% FBS was used as a chemoattractant. Cells $\left(2.5 \times 10^{3}\right)$ were suspended in serum-free DMEM and seeded onto the invasion chambers and control chambers. After $24 \mathrm{~h}$ of incubation, cells were fixed with methanol and stained with crystal violet for $15 \mathrm{~min}$. Cells remaining on the upper face of the membranes were scraped off and those on the lower face were counted using an inverted microscope. All assays were performed in triplicate. The results were calculated by using the following formula: \%Invasion $=($ mean count of invading cells $) /($ mean count of migrating cells) x100.

Statistical analysis. Statistical comparisons of data sets were analyzed by one-way factorial ANOVA and post-hoc test (Dunnett). Data are shown as means \pm standard error of the mean (SEM). These analyses were performed using JMP

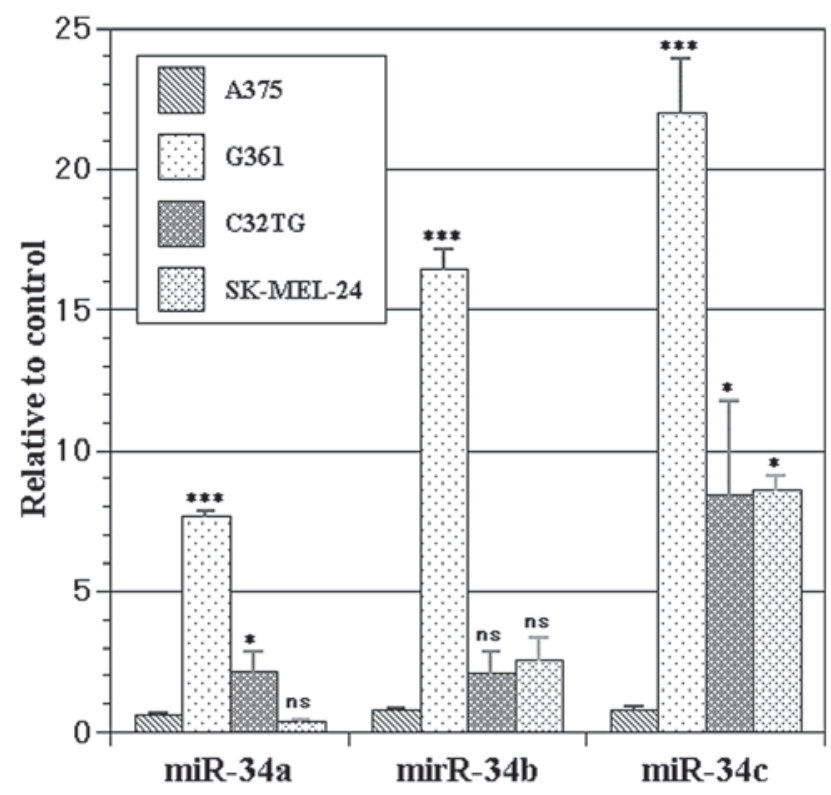

Figure 1. Gene expression levels of miR-34a/b/c evaluated by real-time PCR. Values were normalized by the respective amounts of RNU6B expression as an endogenous control. There was a significant difference among the four cell lines ( $n=4, p<0.0001$, ANOVA). Dunnett's post-hoc test showed significant differences against A375 $\left({ }^{*} \mathrm{p}<0.05,{ }^{* * *} \mathrm{p}<0.001\right)$.

version 8 software (SAS Institute Inc., Cary, NC, USA). P-values $<0.05$ were considered to denote statistical significance.

\section{Results}

Expression of miR-34s in the melanoma cell lines. All four melanoma cell lines showed significant expression of miR-34s as follows - A375: miR-34a 0.6176 \pm 0.0295 , miR-34b $0.7625 \pm 0.0630$, miR-34c $0.7877 \pm 0.1126$; G361: 7.6424 \pm 0.2011 , 16.4127 $\pm 0.7376,22.0332 \pm 1.8522$; C32TG: $2.1630 \pm 0.7064$, $2.1091 \pm 0.7209,8.4425 \pm 3.3104$; SK-MEL-24: $0.3621 \pm 0.0559$, $2.5659 \pm 0.7612,8.5907 \pm 0.5193$ (Fig. 1). Significant differences were noted in the expression of each microRNA (ANOVA, $\mathrm{p}<0.0001$ in miR-34a/b/c). Dunnett's post-hoc test against A375 revealed significant overexpression of miR-34a in G361 $(\mathrm{p}<0.001)$ and C32TG ( $\mathrm{p}=0.0302)$, that of miR-34b in G361 $(\mathrm{p}<0.001)$, and that of miR-34c in G361 $(\mathrm{p}<0.001), \mathrm{C} 32 \mathrm{TG}$ $(\mathrm{p}=0.0387)$ and SK-MEL-24 cells $(\mathrm{p}=0.0351)$.

Growth characteristics of malignant melanoma cell lines. We examined the growth characteristics of the four cell lines in cell culture conditions (Fig. 2). Their cell doubling times were as follows: A375 23:23, G361 68:24, C32TG 47:22 and SK-MEL-24 67:03 (values shown are h:min).

In vitro invasion/migration assays. To investigate the invasive ability of the four cell lines, Matrigel ${ }^{\mathrm{TM}}$ invasion assays were performed (Fig. 3). The percent invasion of each cell line was as follows: A375, 44.00 $\pm 6.52 \%$; G361, 22.37 $\pm 2.71 \%$; C32TG, $13.76 \pm 2.75 \%$; SK-MEL-24, $45.05 \pm 6.71 \%$. These results revealed significant differences (ANOVA, $\mathrm{p}=0.005$ ). Dunnett's post-hoc test against $\mathrm{A} 375$ showed significant differences in G361 ( $\mathrm{p}=0.0405)$ and C32TG ( $=0.0074)$. 


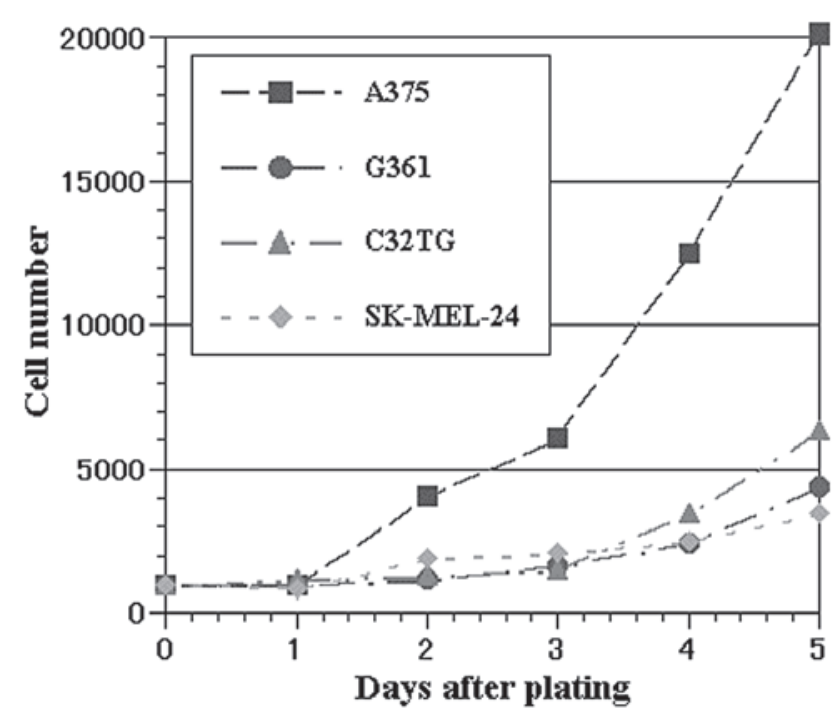

Figure 2. Cell proliferation assay. Cells were seeded at $1.0 \times 10^{3}$ cells/well in 6-well plates. The cell doubling times of these cell lines were as follows: A375 23:23, G361 68:24, C32TG 47:22 and SK-MEL-24 67:03 (values shown are $\mathrm{h}: \mathrm{min})$

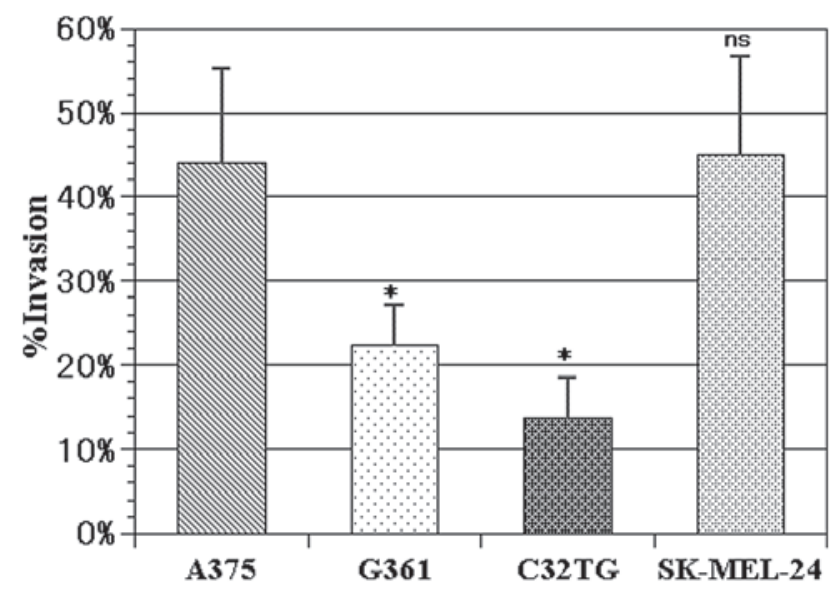

Figure 3. In vitro invasion/migration assays. There was a significant difference among the four cell lines $(n=3, p=0.005$, ANOVA). Dunnett's post-hoc test showed significant differences between A375 and G361 and C32TG $(* \mathrm{p}<0.05)$.

\section{Discussion}

In the present study, we examined the biological role of the miR-34 family in four human malignant melanoma cell lines (A375, G361, C32TG, SK-MEL-24). Real-time PCR revealed that all four melanoma cell lines showed significant expression of the miR-34s, although expression levels of all miR-34s in the A375 cell line were extremely low. Compared with A375, the expression levels of miR-34a in G361 and C32TG cells, miR-34b in the G361 cells, and miR-34c in the G361, C32TG and SK-MEL-24 cell lines were significantly high. The proliferative ability of A375 was higher than that of the other three cell lines. The in vitro invasiveness of A375 and SK-MEL-24 was greater than that of the G361 and C32TG cell lines.
These results suggest that overexpression of miR-34a and c suppresses the invasive and generative potential, respectively, of human malignant melanoma.

p53 is activated by the deregulated expression of oncogenes, which induce replication stress and thereby DNA damage (11). Apart from the direct repressive effects of p53 on core promoters, the induction of microRNAs represents an attractive mechanism for the downregulation of proteins observed after p53 activation (12). MicroRNAs form a class of endogenously expressed, small non-coding RNAs with a recently established key role in the post-transcriptional regulation of gene expression (13-15). microRNA family miR-34s, known for their role in the p53 tumor-suppressor network, are controlled in a tissue-specific manner by p53 directly, inducing apoptosis, cell cycle arrest and senescence $(1,2,16-24)$. Several target genes of miR-34s have been identified $(18,25,26)$. The miR-34 family comprises three processed microRNAs that are encoded by two different genes: miR-34a is encoded by its own transcript, whereas miR-34b and miR-34c share a common primary transcript (1).

In malignant melanoma, the interplay between miR-137 and miR-182 was reported to play a key role in the MITF (microphthalmia-associated transcription factor) regulating network, resulting in degradation of the extracellular matrix and controlling migration/invasion ability $(3,27,28)$. However, Bemis et al suggested that there may be more microRNAs regulating MITF (27). Lodygin et al showed that expression of miR-34a is silenced in various tumors including malignant melanoma due to aberrant $\mathrm{CpG}$ methylation of the corresponding promoter region (29). Migliore et al demonstrated that reduced expression of miR-34b or miR-34c represents an additional pathway for regulating the expression of the MET oncogene in melanocytic cells (30). It was also reported that miR-34a regulates uveal melanoma cell migration through its target gene, c-Met (31).

Ectopic expression of miR-34a was found to cause cell cycle arrest in the G1 phase $(16,19,21)$. In human colon cancer cells, tumor cells showed signs of senescence after introduction of ectopic miR-34a (22). It is also suggested that miR-34a inhibits cell growth and enhances chemosensitivity, as well as cell cycle and apoptosis regulators, in prostate cancer cell lines (32). miR-34b/c, which is also induced by p53, was able to regulate $\mathrm{CpG}$ methylation in oral squamous cell carcinoma and colorectal carcinoma $(33,34)$. It was also reported that miR-34b and c represent novel effectors mediating suppression of such critical components of neoplastic growth as cell proliferation and adhesion-independent colony formation of neoplastic epithelial ovarian cells (18). In this study, miR-34a was inversely correlated with invasiveness, and miR-34c reduced the proliferative potential. Many reports have shown that miR-34b and c have similar biological characteristics. Our results showed that expression levels of $\mathrm{miR}-34 \mathrm{~b} / \mathrm{c}$ were similar to those in other studies, although statistical differences were larger in miR-34c than b. Consequently, there have been many hypotheses regarding the function of $\mathrm{miR}-34 \mathrm{a} / \mathrm{b} / \mathrm{c}$ in various tumor types. The biological characteristics of miR-34a/b/c closely resemble each other, but are by no means identical; they are suggested to interact closely and overlap with each other.

We showed herein that the miR-34 family reduced the tumorigenesis of malignant melanoma, although the detailed 
mechanisms are still unclear. However, there is potential to develop new therapeutic approaches based on microRNA biology. These methods are expected to improve the notably poor prognosis and complete lack of effective standard therapies for malignant melanoma.

\section{References}

1. Hermeking H: p53 enters the microRNA world. Cancer Cell 12: 414-418, 2007.

2. Fabbri M, Croce CM and Calin GA: MicroRNAs. Cancer J 14: $1-6,2008$.

3. Mueller DW and Bosserhoff AK: Role of miRNAs in the progression of malignant melanoma. Br J Cancer 101: 551-556, 2009.

4. Yamashita T, Tokino T, Tonoki $\mathrm{H}$, et al: Induction of apoptosis in melanoma cell lines by p53 and its related proteins. J Invest Dermatol 117: 914-919, 2001.

5. Min FL, Zhang H, Li WJ, Gao QX and Zhou GM: Effect of exogenous wild-type p53 on melanoma cell death pathways induced by irradiation at different linear energy transfer. In Vitro Cell Dev Biol Anim 41: 284-288, 2005.

6. Miyato Y and Ando K: Apoptosis of human melanoma cells by a combination of lonidamine and radiation. J Radiat Res (Tokyo) 45: 189-194, 2004.

7. Monney L, Sabatos CA, Gaglia JL, et al: Th1-specific cell surface protein Tim-3 regulates macrophage activation and severity of an autoimmune disease. Nature 415: 536-541, 2002.

8. Schmittgen TD and Livak KJ: Analyzing real-time PCR data by the comparative C(T) method. Nat Protoc 3: 1101-1108, 2008.

9. Chijiwa T, Abe Y, Ikoma N, et al: Thrombospondin 2 inhibits metastasis of human malignant melanoma through microenvironment-modification in NOD/SCID $/ \gamma$ Cnull (NOG) mice. Int $\mathrm{J}$ Oncol 34: 5-13, 2009.

10. Ikoma N, Yamazaki H, Abe Y, et al: S100A4 expression with reduced $\mathrm{E}$-cadherin expression predicts distant metastasis of human malignant melanoma cell lines in the NOD $/ \mathrm{SCID} / \gamma$ Cnull (NOG) mouse model. Oncol Rep 14: 633-637, 2005.

11. Dominguez-SolaD, Ying CY,GrandoriC, et al: Non-transcriptional control of DNA replication by c-Myc. Nature 448: 445-451, 2007.

12. Ho J and Benchimol S: Transcriptional repression mediated by the p53 tumour suppressor. Cell Death Differ 10: 404-408, 2003

13. Chen K and Rajewsky N: The evolution of gene regulation by transcription factors and microRNAs. Nat Rev Genet 8: 93-103, 2007.

14. Kloosterman WP and Plasterk RH: The diverse functions of microRNAs in animal development and disease. Dev Cell 11: 441-450, 2006

15. Valencia-Sanchez MA, Liu J, Hannon GJ and Parker R: Control of translation and mRNA degradation by miRNAs and siRNAs. Genes Dev 20: 515-524, 2006.

16. Bommer GT, Gerin I, Feng Y, et al: p53-mediated activation of miRNA34 candidate tumor-suppressor genes. Curr Biol 17: 1298-1307, 2007.

17. Chang TC, Wentzel EA, Kent OA, et al: Transactivation of miR-34a by $\mathrm{p} 53$ broadly influences gene expression and promotes apoptosis. Mol Cell 26: 745-752, 2007.
18. Corney DC, Flesken-Nikitin A, Godwin AK, Wang W and Nikitin AY: MicroRNA-34b and microRNA-34c are targets of p53 and cooperate in control of cell proliferation and adhesionindependent growth. Cancer Res 67: 8433-8438, 2007.

19. He L, He X, Lim LP, et al: A microRNA component of the p53 tumour suppressor network. Nature 447: 1130-1134, 2007.

20. Raver-Shapira N, Marciano E, Meiri E, et al: Transcriptional activation of miR-34a contributes to p53-mediated apoptosis. Mol Cell 26: 731-743, 2007.

21. Tarasov V, Jung P, Verdoodt B, et al: Differential regulation of microRNAs by 533 revealed by massively parallel sequencing: miR-34a is a p53 target that induces apoptosis and G1-arrest. Cell Cycle 6: 1586-1593, 2007.

22. Tazawa H, Tsuchiya N, Izumiya $M$ and Nakagama $H$ : Tumorsuppressive miR-34a induces senescence-like growth arrest through modulation of the E2F pathway in human colon cancer cells. Proc Natl Acad Sci USA 104: 15472-15477, 2007.

23. Bagchi A and Mills AA: The quest for the $1 \mathrm{p} 36$ tumor suppressor. Cancer Res 68: 2551-2556, 2008.

24. Zhou Z, Flesken-Nikitin A, Corney DC, et al: Synergy of p53 and $\mathrm{Rb}$ deficiency in a conditional mouse model for metastatic prostate cancer. Cancer Res 66: 7889-7898, 2006.

25. Sun F, Fu H, Liu Q, et al: Downregulation of CCND1 and CDK6 by miR-34a induces cell cycle arrest. FEBS Lett 582: 1564-1568, 2008.

26. Pigazzi M, Manara E, Baron E and Basso G: miR-34b targets cyclic AMP-responsive element binding protein in acute myeloid leukemia. Cancer Res 69: 2471-2478, 2009.

27. Bemis LT, Chen R, Amato CM, et al: MicroRNA-137 targets microphthalmia-associated transcription factor in melanoma cell lines. Cancer Res 68: 1362-1368, 2008.

28. Segura MF, Hanniford D, Menendez S, et al: Aberrant miR-182 expression promotes melanoma metastasis by repressing FOXO3 and microphthalmia-associated transcription factor. Proc Natl Acad Sci USA 106: 1814-1819, 2009.

29. Lodygin D, Tarasov V, Epanchintsev A, et al: Inactivation of miR-34a by aberrant CpG methylation in multiple types of cancer. Cell Cycle 7: 2591-2600, 2008.

30. Migliore C, Petrelli A, Ghiso E, et al: MicroRNAs impair MET-mediated invasive growth. Cancer Res 68: 10128-10136, 2008.

31. Yan D, Zhou X, Chen X, et al: MicroRNA-34a inhibits uveal melanoma cell proliferation and migration through downregulation of c-Met. Invest Ophthalmol Vis Sci 50: 1559-1565, 2009.

32. Fujita Y, Kojima K, Hamada N, et al: Effects of miR-34a on cell growth and chemoresistance in prostate cancer PC3 cells. Biochem Biophys Res Commun 377: 114-119, 2008.

33. Kozaki K, Imoto I, Mogi S, Omura K and Inazawa J: Exploration of tumor-suppressive microRNAs silenced by DNA hypermethylation in oral cancer. Cancer Res 68: 2094-2105, 2008.

34. Toyota M, Suzuki H, Sasaki Y, et al: Epigenetic silencing of microRNA-34b/c and B-cell translocation gene 4 is associated with $\mathrm{CpG}$ island methylation in colorectal cancer. Cancer Res 68: 4123-4132, 2008. 